\title{
Normal galaxies in the XMM-Newton fields
}

\section{X-rays as a star formation indicator ${ }^{\star}$}

\author{
E. Rovilos ${ }^{1,2,3}$, I. Georgantopoulos ${ }^{2}$, P. Tzanavaris ${ }^{4,5,2}$, M. Pracy ${ }^{6}$, M. Whiting ${ }^{7}$, D. Woods ${ }^{8,9}$, and C. Goudis ${ }^{2,3}$ \\ 1 Max Planck Institut für extraterrestrische Physik, Giessenbachstraße, 85748, Garching, Germany \\ e-mail: erovilos@mpe.mpg.de \\ 2 Institute for Astronomy and Astrophysics, National Observatory of Athens, I. Metaxa \& V. Pavlou str, Palaia Penteli, 15236, Greece \\ 3 Astronomical Laboratory, Department of Physics, University of Patras, 26500, Rio-Patras, Greece \\ ${ }^{4}$ Laboratory for X-ray Astrophysics, NASA Goddard Space Flight Center, Mail Code 662, Greenbelt, MD 20771, USA \\ 5 Department of Physics and Astronomy, The Johns Hopkins University, 366 Bloomberg Center, 3400 N. Charles street, Baltimore, \\ MD 21218, USA \\ ${ }^{6}$ Research School of Astronomy and Astrophysics, Mount Stromlo Observatory, Cotter Rd, Weston, ACT 2611, Australia \\ 7 Australia Telescope National Facility, PO Box 76, Epping, NSW 1710, Australia \\ 8 School of Physics, University of New South Wales, Sydney NSW 2052, Australia \\ 9 Dept. of Physics \& Astronomy, University of British Columbia, 6224 Agricultural Road, Vancouver B.C., V6T 1Z1, Canada
}

Received 12 March 2009 / Accepted 18 May 2009

\section{ABSTRACT}

Context. We use the first XMM serendipitous source catalogue (1XMM) to compile a sample of normal X-ray galaxies. Aims. We seek to expand the database of X-ray selected normal galaxies at intermediate redshifts and examine the relation between $\mathrm{X}$-ray emission and star formation for late-type systems.

Methods. The candidates are selected based on their X-ray (soft spectra), X-ray to optical $\left(\log \left(f_{\mathrm{x}} / f_{\mathrm{o}}\right)<-2\right)$ and optical (extended sources) properties. 44 candidates are found and 35 are spectroscopically observed with the Australian National University's $2.3 \mathrm{~m}$ telescope to examine their nature.

Results. Of the 35 sources observed, 2 are AGN, 11 emission line galaxies, 12 absorption line galaxies, 6 have featureless spectra while 4 are associated with Galactic stars. We combine our emission line sample with earlier works forming the most comprehensive $\mathrm{X}$-ray selected galaxy sample for the study of the X-ray luminosity to the $\mathrm{H} \alpha$ luminosity - a well-calibrated star-formation indicator - relation.

Conclusions. We find that the X-ray luminosity strongly correlates with the $\mathrm{H} \alpha$ luminosity, suggesting that the X-rays efficiently trace the star-formation.

Key words. galaxies: starburst - X-rays: galaxies

\section{Introduction}

$\mathrm{X}$-ray emission from normal galaxies (i.e. galaxies which do not host an AGN) has been targeted by X-ray telescopes since the early years of X-ray astronomy. Normal galaxies are generally divided into two broad morphological categories, latetype (spirals and irregulars) and early-type (S0 and ellipticals). Early studies of optically selected samples of early-type galaxies with EINSTEIN (Trinchieri \& Fabbiano 1985; Fabbiano et al. 1987) have examined the relation between the X-ray, the blue optical and radio luminosities and argued that the X-ray luminosity arises from a combination of low-mass X-ray binaries (LMXRBs) and hot gas (e.g. Forman et al. 1979). On the other hand, the X-ray study of late-type systems with EINSTEIN (Fabbiano \& Trinchieri 1985; Fabbiano et al. 1988) has revealed a different kind of X-ray correlation for the radio (shallower) and blue (more linear) luminosities, as well as a correlation between $\mathrm{X}$-ray and infrared luminosity, suggesting a link with on-going star formation or starburst activity. Optically selected samples of normal galaxies observed with ROSAT generally confirmed the

\footnotetext{
* Table 2 and Figures 1 and 3 are only available in electronic form at http://www. aanda.org
}

above picture. Studying the X-ray emission of spiral galaxies and analyzing its compact and diffuse components, Read et al. (1997) have shown that its origins are hot diffuse continuum, supernova remnants and high-mass X-ray binaries (HMXRBs), phenomena which are related to star formation activity, and that their relative strength is also related with the galaxy's activity (Read \& Ponman 2001).

The above studies are restricted to optically selected samples of normal galaxies in the nearby universe. The advent of the second-generation X-ray telescopes (Chandra and XMM) has allowed for the first time the compilation of X-ray selected normal galaxy samples. Their selection is usually based on low X-ray to optical flux ratios (see Hornschemeier et al. 2003) and optical spectral properties (e.g. Norman et al. 2004; Bauer et al. 2004). Searches for normal galaxies in broad (e.g. Georgakakis et al. 2003, 2004, 2006; Hornschemeier et al. 2005; Tajer et al. 2005; Tzanavaris et al. 2006) and deep (e.g. Alexander et al. 2002; Hornschemeier et al. 2003; Norman et al. 2004; Georgakakis et al. 2007) surveys have provided a large number of X-ray selected galaxies. Using these samples, the luminosity function of normal galaxies has been established, both locally (e.g. Georgantopoulos et al. 2005; Georgakakis et al. 2006) and at 
higher redshifts (e.g. Norman et al. 2004; Ptak et al. 2007; Tzanavaris \& Georgantopoulos 2008).

One of the most interesting properties of the X-ray emission of normal galaxies is its connection to star formation. New results have confirmed earlier findings that X-rays can act as a star formation indicator in late-type systems. The tight correlation of the X-ray luminosity with other star formation indicators, such as infrared and radio luminosities (Shapley et al. 2001; Ranalli et al. 2003) support this hypothesis. The X-ray emission of late-type galaxies comes from both their extended structures (spiral arms and the Galactic centre) and compact sources. Emission from the spiral arms is tightly connected with star formation (Tyler et al. 2004), while the connection to the Galactic centre is less well defined. The compact sources on the other hand are mostly HMXRBs (Colbert et al. 2004) which are directly connected to star formation as end products of massive rapidly evolving stars (Persic et al. 2004; Persic \& Rephaeli 2007). Grimm et al. (2003) and Gilfanov et al. (2004) reproduce the observational characteristics of X-ray normal galaxies as a combination of discrete point sources and predict a steep ( $L_{\mathrm{x}} \propto \mathrm{SFR}^{\beta}$ with $\beta>1$ ) $L_{\mathrm{x}}-\mathrm{SFR}$ relation when the star formation rate is low $\left(\lesssim 4.5 M_{\odot} \mathrm{yr}^{-1}\right)$ and a linear $(\beta=1)$ relation thereafter.

In this paper we expand the galaxy samples of Georgakakis et al. (2006) and explore the relation between the X-ray and $\mathrm{H} \alpha$ luminosities for emission line systems. This provides an insight into the $L_{\mathrm{x}}-\mathrm{SFR}$ relation (see also Hornschemeier et al. 2005).

\section{Sample selection}

The first XMM Newton Serendipitous Source Catalogue (1XMM, Watson et al. 2003) is a compilation of source detections drawn from 585 XMM-Newton EPIC observations made between March 2000 and May 2002, and released in January 2003. It contains $\sim 55000$ sources, of which $\sim 33000$ are considered safe detections and covers an area of $\sim 90 \mathrm{deg}^{2}$. From the 1XMM catalogue we selected only sources which are observed for more than $10 \mathrm{ks}$ to achieve a reasonable signal-to-noise ratio in X-rays and required the detection likelihood to be above $10 \sigma$ in the soft band $(0.5-2.0 \mathrm{keV})$. Since we are aiming for normal galaxies, we limited our search sample to sources with hardness ratios $<-0.2$ (between the $0.5-2.0$ and $2.0-4.5 \mathrm{keV}$ bands) to avoid X-ray obscured AGN. This limit is unlikely to reject any normal galaxies since their hardness ratios are normally $<-0.2$ (see e.g. Table 1 of Tzanavaris et al. 2006).

To identify these X-ray sources with optical counterparts, we used the USNO-B optical catalogue (Monet et al. 2003). This is an all-sky catalogue of $\sim 10^{9}$ optical sources observed during various surveys over the past 50 years. We searched for optical counterparts to the X-ray sources in the USNO-B catalogue with a search radius of 6 arcsec (see Fig. 1). We limited our search to the southern hemisphere $(\delta<0)$ in order to be within the observing range of the Siding Spring Observatory, and to avoid Galactic stars and high Galactic $N_{\mathrm{H}}$ we excluded sources that are within a belt of \pm 20 degrees of Galactic latitude. We also avoided the areas of the sky close to the Magellanic Clouds, with an exclusion radius of 3 and 1.5 degrees from the centres of the LMC and the SMC respectively. This left us with an initial catalogue of $\sim 16000$ XMM sources, of which 4700 are associated with an optical detection.

A useful diagnostic to discriminate between normal galaxies and AGN is the X-ray to optical flux ratio $\left(f_{\mathrm{x}} / f_{\mathrm{o}}\right)$. Hornschemeier et al. (2003) found that optically bright $\mathrm{X}$-ray faint objects (OBXF, which they define as having $\left.\log \left(f_{\mathrm{x}} / f_{\mathrm{o}}\right)<-2.3\right)$ are almost exclusively galaxies with a contamination from stars. Moreover, AGN are usually confined in the area $-1<\log \left(f_{\mathrm{x}} / f_{\mathrm{o}}\right)<1$ (Stocke et al. 1991; Lehmann et al. 2001). For the purposes of this study we selected as normal galaxy candidates sources having $\log \left(f_{\mathrm{x}} / f_{\mathrm{o}}\right)<-2$ (see also Georgakakis et al. 2004; Georgantopoulos et al. 2005; Georgakakis et al. 2006). This is a conservative limit and it might introduce a bias against massive ellipticals and powerful starbursts (Tzanavaris et al. 2006; Georgakakis et al. 2007). On the other hand it minimizes contamination from AGN with low X-ray luminosities having unusually low $f_{\mathrm{x}} / f_{\mathrm{o}}$ (Lehmann et al. 2001). To calculate the X-ray to optical flux ratio we used:

$\log \left(\frac{f_{\mathrm{x}}}{f_{\text {opt }}}\right)=\log f_{\mathrm{x}}+\frac{R}{2.5}+5.5$

(e.g. Hornschemeier et al. 2003), where $f_{\mathrm{x}}$ is the $(0.5-2.0) \mathrm{keV}$ $\mathrm{X}$-ray flux in $\mathrm{erg} \mathrm{s}^{-1} \mathrm{~cm}^{-2}$, and $R$ is the magnitude from the USNO-B catalogue.

Selecting our sources taking into account the hardness ratio and the X-ray to optical flux ratio, left us with a catalogue of 713 normal galaxy candidates contaminated with a large number of Galactic stars, as they have similar observational characteristics. We used optical morphologies to identify and distinguish the stars from the galaxies. For that purpose, we used the APM Sky Catalogues ${ }^{1}$ (Irwin et al. 1994), which are a digitized compilation of the Palomar $\mathrm{O}$ and $\mathrm{E}$ sky survey in the northern and the UKST $B_{\mathrm{J}}$ sky survey in the southern hemisphere. They also include a star-galaxy separation parameter based on the extended nature of each source, which we used to select extragalactic candidates. Visual inspection of the sources with the DSS2-Red image (Lasker et al. 1996) generally agrees with the star-galaxy separation from the APM; $98 \%$ of sources characterized as stars with APM are independently characterized as stars when optically inspecting the images.

The final catalogue consists of 44 normal galaxy candidates, listed in Table 2. Their DSS2-Red optical images with an indication on the location of the X-ray source are shown in Fig. 1. The $\mathrm{X}$-ray fluxes of the sources are calculated from the $0.5-2 \mathrm{keV}$ count rates given in the $1 \mathrm{XMM}$ catalogue, assuming a powerlaw X-ray spectrum with $\Gamma=1.9$. In Fig. 2 we plot the $R$ magnitude with respect to the $\mathrm{X}$-ray flux for emission and absorption line galaxies. We do not observe any difference between early and late-type galaxies in terms of their relative X-ray to optical fluxes.

\section{Observations and data reduction}

Long-slit spectral observations were carried out at the Australian National University's $2.3 \mathrm{~m}$ telescope in Siding Spring, Australia, between 2006 October 23-29. We used the Double Beam Spectrograph (DBS) with dichroic \#3 and 600 lines/mm gratings. We set the central wavelength at $5100 \AA$ (7000 $\AA$ ) in the blue (red) arm, thus obtaining continuous coverage over the spectral region $4140 \AA-7965 \AA$ with a sampling of $\sim 2 \AA /$ pixel and with a $1^{\prime \prime}$ slit. As our observing run was partly affected by cloudy conditions, we were only able to observe 35 of the full set of 44 normal galaxy candidates. Standard calibration exposures were carried out, including comparison lamps and spectrophotometric standard stars for wavelength and flux calibration, respectively.

1 http://www.ast.cam.ac.uk/ mike/apmcat/ 


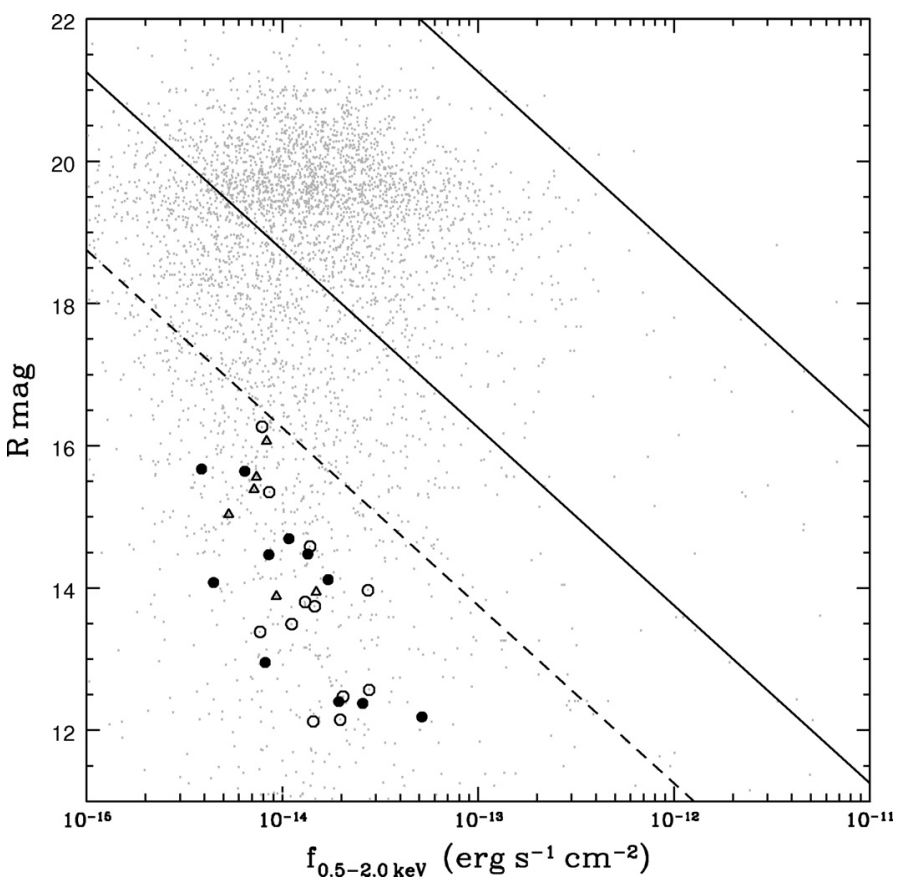

Fig. 2. $R$-band magnitude versus $\mathrm{X}$-ray flux for emission and absorption line galaxies in our sample, marked with filled and open circles respectively. Open triangles mark sources with featureless spectra. Solid lines represent $f_{\mathrm{x}} / f_{\mathrm{o}}=1$ and -1 , where the bulk of AGN are expected, and the dashed line represents $f_{\mathrm{x}} / f_{\mathrm{o}}=-2$, our limit for normal galaxy selection. Background dots represent 1XMM sources not in our sample.

Standard IRAF $^{2}$ routines were used for data reduction. All frames were overscan and bias-subtracted. Observations at the same setting were combined to increase the signal-tonoise ratio $(S / N)$ and to remove cosmic rays. Subsequently, the spectra were flat-fielded, sky-subtracted, wavelength- and fluxcalibrated. Details of the observations can be found in Table 1. The signal-to-noise ratio is the ratio of the most prominent feature of each spectrum, which for emission-line systems is the $\mathrm{H} \alpha$ line and for absorption-line systems the $\mathrm{Na} \mathrm{D}$ doublet.

\section{Optical spectral properties}

We have obtained spectra for 35 of the normal galaxy candidates, which are shown in Fig. 3. For 29 sources we were able to identify emission and absorption lines, the most prominent of which are: $\mathrm{H} \alpha, \mathrm{H} \beta, \mathrm{H} \gamma,[\mathrm{S} \mathrm{II}](6718 \AA),[\mathrm{S} \mathrm{II}](6731 \AA)$, [O I] $(6300 \AA),[\mathrm{O} \mathrm{III}](4959 \AA),[\mathrm{O}$ III] $(5007 \AA),[\mathrm{N} \mathrm{II}](6548 \AA)$, [N II] $(6584 \AA)$ in emission, and Na D1 (5898 $), \mathrm{Na} \mathrm{D} 2(5892 \AA)$, Mg I b2(5172 A), Fe G(4308 ̊), Ca HK(3934 \& $3968 \AA)$ in absorption. 11 galaxies are characterized as emission line systems, 12 absorption line systems, while 2 sources show both emission and absorption lines and are characterized as composite and 4 sources have stellar-like spectra. The redshifts of the sources with identified lines were calculated from the rest-frame frequencies by fitting Gaussians to the line profiles. They agree with published values. 7 of the redshifts we obtained are not published in the literature.

In cases of systems with emission lines, we measured their intensities and used the line ratios to trace signs of AGN. For

${ }^{2}$ IRAF is distributed by the National Optical Astronomy Observatories, which is operated by the Association of Universities for Research in Astronomy, Inc. (AURA) under cooperative agreement with the National Science Foundation.
Table 1. Observation summary.

\begin{tabular}{|c|c|c|c|c|}
\hline ID & XMM name & Obs.date & $\begin{array}{l}\text { Exp. time } \\
\text { mn }\end{array}$ & $S / N$ \\
\hline 1703 & J005818.3-355548 & Oct. 232006 & 60 & 32.1 \\
\hline 1774 & J005922.8-360933 & Oct. 262006 & 45 & 3.6 \\
\hline 1778 & J005929.7-361113 & Oct. 282006 & 30 & 34.0 \\
\hline 3654 & J022416.7-050323 & Oct. 292006 & 55 & - \\
\hline 3716 & J022456.2-050801 & Oct. 242006 & 40 & 2.5 \\
\hline 3773 & J022536.4-050012 & Oct. 242006 & 30 & 84.7 \\
\hline 3776 & J022537.8-050223 & Oct. 242006 & 40 & - \\
\hline 3777 & J022538.2-050806 & Oct. 252006 & 45 & 118.0 \\
\hline 3778 & J022538.3-050423 & Oct. 292006 & 30 & 3.4 \\
\hline 4009 & J023613.5-523036 & Oct. 232006 & 40 & 131.4 \\
\hline 4298 & J030927.5-765223 & Oct. 282006 & 40 & 4.5 \\
\hline 4379 & J031256.5-765039 & Oct. 292006 & 30 & 9.4 \\
\hline 4481 & J031723.1-442056 & Oct. 282006 & 40 & 4.5 \\
\hline 4736 & J031829.8-441140 & Oct. 292006 & 45 & 23.7 \\
\hline 4765 & J031845.0-441042 & Oct. 232006 & 55 & 11.4 \\
\hline 4927 & J033831.4-351421 & Oct. 252006 & 40 & 6.8 \\
\hline 5570 & J043306.5-610760 & Oct. 242006 & 55 & 3.3 \\
\hline 5663 & J043333.5-612427 & Oct. 242006 & 40 & 2.3 \\
\hline 7355 & J055940.7-503218 & Oct. 242006 & 45 & 2.7 \\
\hline 7400 & J060014.9-502230 & Oct. 242006 & 30 & - \\
\hline 12281 & J201329.7-414737 & Oct. 272006 & 45 & 79.2 \\
\hline 12308 & J201345.0-563713 & Oct. 232006 & 30 & $2.0^{1}$ \\
\hline 13137 & J213758.7-143611 & Oct. 232006 & 30 & 61.6 \\
\hline 14402 & $\mathrm{~J} 221726.0-082531$ & Oct. 262006 & 30 & 2.6 \\
\hline 14541 & J222110.0-244749 & Oct. 282006 & 40 & - \\
\hline 14608 & $\mathrm{~J} 222804.4-051751$ & Oct. 292006 & 45 & - \\
\hline 15022 & J225149.3-175225 & Oct. 232006 & 30 & 153.7 \\
\hline 15393 & J231421.6-424559 & Oct. 242006 & 40 & 4.0 \\
\hline 15584 & $\mathrm{~J} 231851.8-423114$ & Oct. 252006 & 30 & 2.9 \\
\hline 15631 & J232454.9-120459 & Oct. 282006 & 45 & 1.9 \\
\hline 15867 & $\mathrm{~J} 235340.6-102420$ & Oct. 232006 & 30 & 3.2 \\
\hline 15892 & J235405.7-101829 & Oct. 272006 & 45 & 1.3 \\
\hline 15926 & J235418.1-102013 & Oct. 282006 & 40 & 14.3 \\
\hline 15997 & J235629.1-343743 & Oct. 252006 & 30 & $2.0^{1}$ \\
\hline
\end{tabular}

$S / N$ of the $\mathrm{Mg}$ I b2 line.

this purpose, we use the line ratio diagnostics of Ho et al. (1997), which are based on the ratios of the [O III](5007 $\AA$ ), [O I] $(6300 \AA),[\mathrm{N} \mathrm{II}](6584 \AA),[\mathrm{S} \mathrm{II}](6718 \AA)$ and [S II] $(6731 \AA)$ lines with the Balmer lines $(\mathrm{H} \alpha$ and $\mathrm{H} \beta)$. These line combinations $([\mathrm{O} \mathrm{III}] / \mathrm{H} \beta-[\mathrm{O} \mathrm{I}] / \mathrm{H} \alpha-[\mathrm{N} \mathrm{III}] / \mathrm{H} \alpha-[\mathrm{S} \mathrm{II}] / \mathrm{H} \alpha)$ are selected to have small wavelength separation and therefore be insensitive to reddening (see also Veilleux \& Osterbrock 1987). Without including any reddening correction, we find that the emission line ratios are compatible with starburst galaxies for all the emission line systems. For systems having composite spectra, the emission line ratios are compatible with Seyfert nuclei. These are removed from our sample of normal galaxies.

Sources which show no prominent features in their spectra are marked as "featureless" in Table 2. A featureless optical spectrum can indicate a BL Lac object (e.g. Londish et al. 2002; Sbarufatti et al. 2006). The broadband spectra of BL Lacs show a variety of X-ray to optical to radio flux ratios (Bondi et al. 2001) and there are cases where BLLacs are bright in optical and faint in X-rays (Troitsky 2008). The host galaxies of BL Lacs are in most cases ellipticals or bulge dominated systems (Falomo 1996) and optical images are consistent with this picture. We therefore cannot rule out that at least some of the sources with featureless spectra harbor AGN and we remove them from our final sample of normal galaxies. 


\section{Starburst diagnostics}

A good diagnostic of the star formation rate is the luminosity of the $\mathrm{H} \alpha$ line, which is found to scale linearly with the SFR for nearby galaxies and also at higher redshifts (Kennicutt et al. 1994; Kewley et al. 2002; Rosa-Gonzàlez et al. 2002). The X-ray versus the $\mathrm{H} \alpha$ luminosity diagram is shown in Fig. 4. We corrected the derived $\mathrm{H} \alpha$ luminosity for dust extinction using the reddening curves of Savage \& Mathis (1979) and the flux ratio of the $\mathrm{H} \alpha$ and $\mathrm{H} \beta$ lines, assuming an intrinsic value of 2.76 (Brocklehurst 1971). Our data points are plotted as open circles in Fig. 4, while crosses represent the combined sample of the 1XMM survey of Georgakakis et al. (2006) and the NHS survey (Georgakakis et al. 2004).

Because our dataset has errors in both directions we choose a fitting method that deals with the two axes in a symmetric way. As such we choose the bisector line and the orthogonal regression method (Isobe et al. 1990), which yield $\beta=0.72 \pm 0.04$ and $\beta=0.68 \pm 0.05$ respectively when fitting $L_{\mathrm{x}}=\alpha L_{\mathrm{H} \alpha}^{\beta}$. These are the solid and dashed lines in Fig. 4. These values lie within the uncertainty ranges of the measurements made by Griffiths \& Padovani (1990) $(0.70 \pm 0.12)$, Zezas (2000) $(0.62 \pm 0.11)$, and Georgakakis et al. (2006) $(0.69 \pm 0.06)$ and they are not linear. If we attribute the X-ray emission to a number of HMXRBs, the relation is expected to be linear (Persic et al. 2004; Persic \& Rephaeli 2007). Indeed, a linear relation is found by David et al. (1992) and Ranalli et al. (2003) for both soft and hard $\mathrm{X}$-rays, using the far-infrared and radio $(1.4 \mathrm{GHz})$ as star formation indicators. Non-linearity is expected in cases of very low or very high star formation rates, where, as a result of small number statistics and hypothetical intermediate mass black holes respectively, it is expected to be even steeper with $\beta>1$ (Grimm et al. 2003; Gilfanov et al. 2004). If we use the relation: $\operatorname{SFR}\left(M_{\odot} \mathrm{yr}^{-1}\right)=7.9 \times 10^{-42} L_{\mathrm{H} \alpha}\left(\mathrm{erg} \mathrm{s}^{-1}\right)$ (Kennicutt et al. 1994), we are expecting the low-SFR cut at $L_{\mathrm{H} \alpha} \sim 10^{42} \mathrm{erg} \mathrm{s}^{-1}$, which is within the range we are sampling (see dotted line in Fig. 4). It is therefore puzzling that the $L_{\mathrm{x}}-L_{\mathrm{SFR}}$ rate appears flatter than linear.

Such a behavior could be explained by the existence of other sources of X-ray emission not connected with star formation activity. As such we could consider LMXRBs, older supernova remnants or globular clusters (see e.g. Vogler et al. 1997) and hot diffuse gas toward the Galactic centre (Tyler et al. 2004). If not linked with star formation, their relative contribution to the total X-ray luminosity would be stronger in galaxies with lower star formation rates, thus flattening the $L_{\mathrm{X}}-L_{\mathrm{SFR}}$ relation.

The combined X-ray luminosity of LMXRBs is associated with the stellar content of the galaxy (Gilfanov 2004), which can be examined from its luminosity in the $K$-band. For that purpose we search for counterparts of the galaxies in our sample in the 2MASS survey catalogues (Skrutskie et al. 2006). We then calculate the $K$-band luminosities and use the mass-to-light ratio of Bell \& de Jong (2001) to derive the stellar mass of each source. This is translated into the respective luminosity of the LMXRB content using the relation of Gilfanov et al. (2004); the results are presented in Table 2. We find that the combined X-ray luminosity of LMXRBs is two orders of magnitude fainter than the total X-ray luminosity and thus not enough to have an effect on the $L_{\mathrm{x}}-L_{\mathrm{SFR}}$ correlation.

In Fig. 5 we plot two different hardness ratios for emission and absorption like systems. HR 1 refers to the hardness ratio between the $(0.5-2.0) \mathrm{keV}$ and $(2.0-4.5) \mathrm{keV}$ bands, whereas HR2 refers to the (2.0-4.5) keV and (4.5-7.5) keV bands. As different sources have been observed with XMM-Newton in different

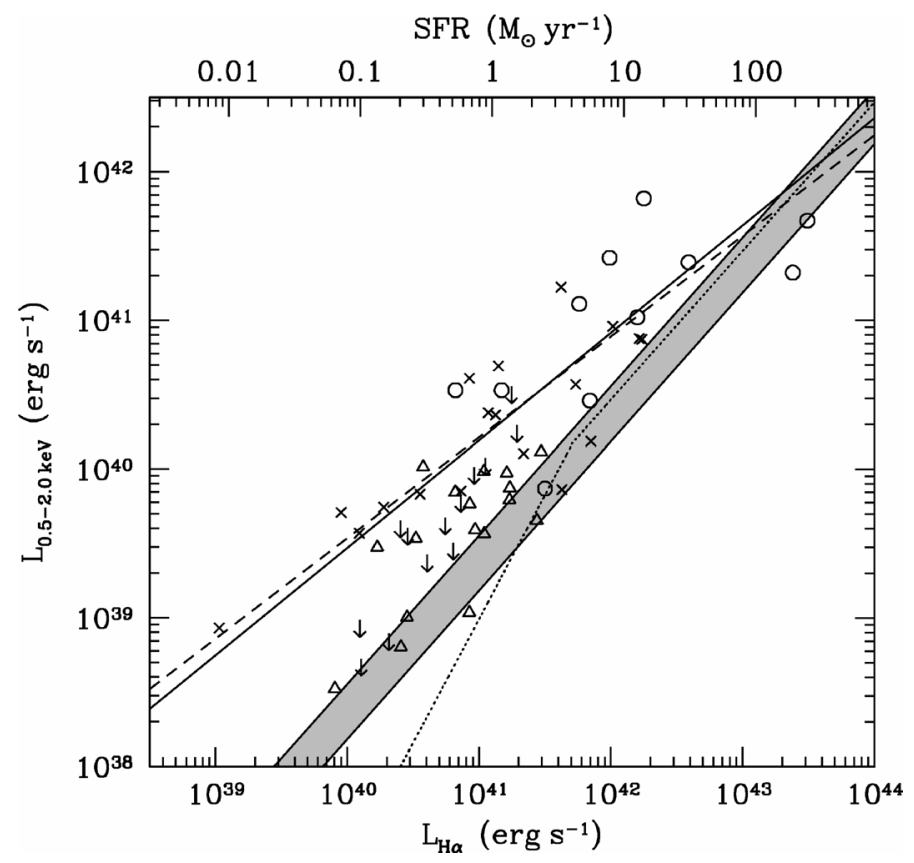

Fig. 4. X-ray luminosity (in the $0.5-2 \mathrm{keV}$ band) plotted against the luminosity of the $\mathrm{H} \alpha$ line for late-type galaxies. With circles are plotted the data-points of this study and with crosses the data-points of Georgakakis et al. (2006). Triangles and arrows represent the datapoints (and respective upper limits) of the combined sample of local spirals from Shapley et al. (2001) and Trinchieri et al. (1989). The solid and dashed lines are the fits to the combined dataset using the bisector line and the orthogonal regression respectively. The shaded region represents the relations of Ranalli et al. (2003) and Persic \& Rephaeli (2007) and the dotted line the relation of Grimm et al. (2003). The SFR scale is also shown for comparison on the top axis.

modes with different CCDs and different optical light blocking filters, to have consistent hardness ratios, we transformed all count rates to the PN CCD with thin filter mode, correcting for the respective galactic absorption of each source. Late-type galaxies are represented by blue colour and early-type by red. The errors in the count rates of each source have been taken into account to create a 2 dimensional Gaussian to be used in creating the colour representation of Fig. 5 (online version).

All sources by selection have HR $1<-0.2$. We can see that the bulk of the late-type galaxies appear soft in both hardness ratios, which is not a typical behavior of HMXRBs, therefore a contribution of another X-ray source should be considered.

In studies of nearby galaxies, where the X-ray emission from point sources can be resolved out, the diffuse emission from hot gas is generally spatially coincident with star formation regions (e.g. Doane et al. 2004; Warwick et al. 2007; Owen \& Warwick 2009), consistent with its heating mechanism being supernova explosions and the winds of very massive stars, possibly with a small contribution from the bulge (Tyler et al. 2004). The origin of it in star forming related properties would make its contribution scale linearly with the star formation rate and not affect the slope of the $L_{\mathrm{x}}-L_{\mathrm{SFR}}$ relation. Persic et al. (2004) find that considering only the X-ray contribution of the point sources in their sample of star forming galaxies, that affects only the scatter of the $L_{\mathrm{x}}-L_{\mathrm{SFR}}$ relation (making it smaller) and not the slope which is in both cases linear.

In order to test the non-linearity of the $L_{\mathrm{x}}-L_{\mathrm{SFR}}$ relation, we attempt to reproduce it using other proxies of star formation rate than the $\mathrm{H} \alpha$ luminosity. The far infrared luminosity is a good 


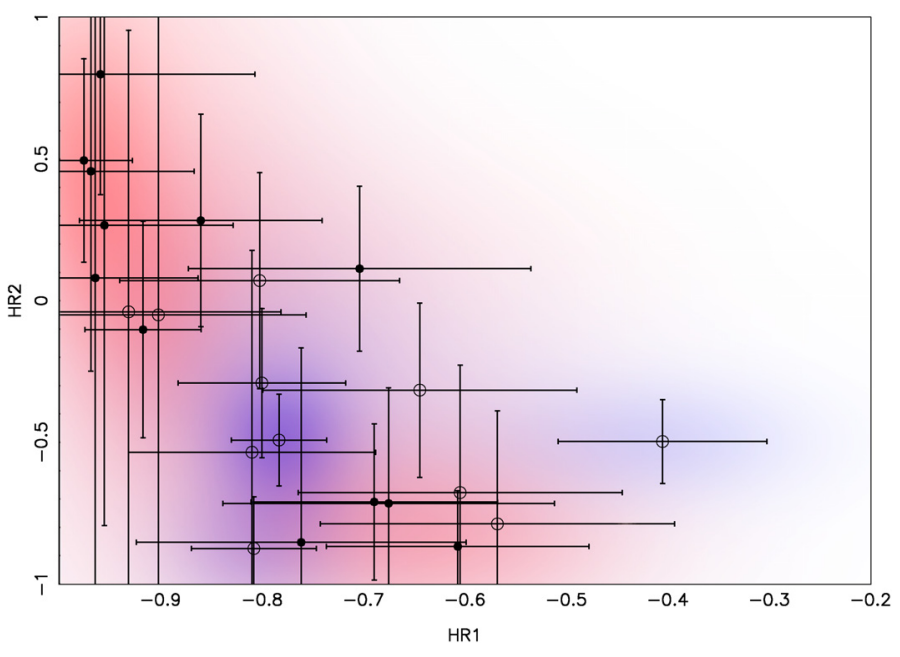

Fig. 5. X-ray colour-colour diagram. HR1 and HR2 are the hardness ratios between the (0.5-2.0) keV and (2.0-4.5) keV, and (2.0-4.5) keV and (4.5-7.5) keV bands respectively. Open circles represent late-type and filled circles early-type galaxies. Online version: Blue and red coloured Gaussians are plotted at each late and early type point respectively, with their standard deviations equal to the uncertainty of the hardness ratio of each point.

example and we search for FIR counterparts of the late-type galaxies in the combined sample of this study and that of Georgakakis et al. (2006) in the IRAS faint source catalogue (Moshir et al. 1990). We detect 8 sources and calculate their FIR luminosities using the formula of Helou et al. (1985). We find tentative evidence that the $L_{\mathrm{x}}-L_{\mathrm{FIR}}$ is flatter than linear ( $b=0.81 \pm 0.09$ and $b=0.76 \pm 0.11$ using again the bisector and the orthogonal regression lines). However the number of data points is too small to allow us to extract conclusive results; moreover earlier studies of the $L_{\mathrm{x}}-L_{\mathrm{FIR}}$ relation using larger samples (e.g. Fabbiano \& Shapley 2002) are in agreement with a linear relation.

We must caution here two effects that might bias our data. Our sample and also these of Georgakakis et al. (2004) and Georgakakis et al. (2006) are selected using an $f_{\mathrm{x}} / f_{\mathrm{o}}$ cut-off and are thus flux limited in the X-rays. A flux limited sample could introduce a bias in the $L_{\mathrm{x}}-L_{\mathrm{SFR}}$ relation flattening it. Also, the star formation rate is proxied by the luminosity of the $\mathrm{H} \alpha$ line. The $\mathrm{H} \alpha$ flux is measured through slit spectroscopy and this technique allows only a fraction of the light of the source to be detected. The slit width is $1^{\prime \prime}$, which corresponds to $3.4 \mathrm{kpc}$ at a redshift of 0.21 and to $0.23 \mathrm{kpc}$ at a redshift of 0.0115 . This is significantly smaller than the typical diameter of a spiral galaxy. Moreover, this effect is more severe for galaxies at lower redshift, which are the less luminous in flux limited surveys. This introduces a bias in the $L_{\mathrm{x}}-L_{\mathrm{SFR}}$ relation and more specifically it flattens it. If we check the normalization of the $L_{\mathrm{x}}-L_{\mathrm{SFR}}$ we derive with respect to Ranalli et al. (2003) and (Persic \& Rephaeli 2007) (shaded region in Fig. 4, see also Hornschemeier et al. 2005 and references therein), we find an agreement for luminous sources (at higher redshifts) and a deviation of fainter sources (at lower redshifts). Interestingly, the normalization in the case of Hornschemeier et al. (2005) where there is a correction applied to the $\mathrm{H} \alpha$ fluxes to compensate for the aperture effect seems to be in good agreement with Ranalli et al. (2003) and Persic \& Rephaeli (2007).

To further investigate the aperture effect to our data, we compile a sample of (nearby) normal galaxies with measured X-ray and integrated $\mathrm{H} \alpha$ luminosities. We use the EINSTEIN spiral galaxy sample of Shapley et al. (2001) and combine it with the sample of Trinchieri et al. (1989), which uses integrated $\mathrm{H} \alpha$ measured from Kennicutt \& Kent (1983). We find 30 common sources between the two samples, not including sources from the Shapley et al. (2001) sample which show evidence for nonstellar ionizing source. We transform the X-ray luminosities to the $0.5-2.0 \mathrm{keV}$ band using a power-law profile with $\Gamma=1.9$. We also adopt the distances from Shapley et al. (2001) and transform the $\mathrm{H} \alpha$ luminosities accordingly. The $L_{\mathrm{x}}-L_{\mathrm{H} \alpha}$ pairs are plotted with triangles in Fig. 4 (arrows indicate upper $L_{\mathrm{x}}$ limits). We can see that these data-points lie close to the shaded area and are systematically more $\mathrm{H} \alpha$ luminous compared to the data-points of this study, and the bisector line fit (not taking upper limits into account) has a slope of $\beta=1.05 \pm 0.10$. We therefore conclude that it the "aperture effect" of slit spectroscopy plays an important role in the flattening of the $L_{\mathrm{x}}-L_{\mathrm{SFR}}$ relation.

\section{Summary and conclusions}

In this study we select normal galaxies from the XMM-Newton first serendipitous source catalogue (1XMM). We select soft sources with reliable detections and compare their X-ray with their optical fluxes. After selecting candidates with $\log \left(f_{\mathrm{x}} / f_{\mathrm{o}}\right)<$ -2 we examine their morphologies to remove any contamination from Galactic stars. This way we are left with a sample of 44 sources (in the southern hemisphere). We observed 35 of them with the ANU's 2.3 m telescope to derive their optical spectra. Of the 35 sources, 4 are Galactic stars, 2 are associated with AGN, and 6 have featureless spectra and are probably associated with BL-Lac objects. The remaining $23(65.7 \%)$ are indeed normal galaxies with emission (11) and absorption-line (12) spectra.

We examined the sub-sample of emission line galaxies (late type) to derive their star formation properties. For this purpose we combined our sample with those of Georgakakis et al. (2004) and Georgakakis et al. (2006). We find tentative evidence that the X-ray luminosity does not scale linearly with star formation, approximated by the $\mathrm{H} \alpha$ luminosity, this result is however sensitive to observational and selection biases. The normalization of the $L_{\mathrm{X}}-L_{\mathrm{SFR}}$ relation agrees with previous studies at the luminous end, indicating underestimation of the $\mathrm{H} \alpha$ luminosities for fainter sources.

Acknowledgements. E.R. wishes to thank the European Social Fund (ESF), Operational Program for Educational and Vocational Training II (EPEAEK II), and particularly the Program PYTHAGORAS II, for funding part of this work.

\section{References}

Alexander, D. M., Aussel, H., Bauer, F. E., et al. 2002, ApJ, 568, L85

Bauer, F. E., Alexander, D. M., Brandt, W. N., et al. 2004, AJ, 128, 2048

Bell, E. F., \& de Jong, R. S. 2001, ApJ, 550, 212

Bondi, M., Marchã, M. J. M., Dallacasa, D., \& Stranghellini, C. 2001, MNRAS, 325,1109

Brocklehurst, M. 1971, MNRAS, 153, 471

Colbert, E. J. M., Heckman, T. M., Ptak, A. F., \& Strickland, D. K. 2004, ApJ, 602,231

David, L. P., Jones, C., \& Forman, W. 1992, ApJ, 388, 82

Doane, N. E., Sanders, W. T., Wilcots, E. M., \& Juda, M. 2004, AJ, 128, 2712

Fabbiano, G., \& Trinchieri, G. 1985, ApJ, 196, 430

Fabbiano, G., \& Shapley, A. 2002, ApJ, 565, 908

Fabbiano, G., Klein, U., Trinchieri, G., \& Wielebinski, R. 1987, ApJ, 111, 121

Fabbiano, G., Gioia, I. M., \& Trinchieri, G. 1988, ApJ, 324, 749

Falomo, R. 1996, MNRAS, 283, 241

Forman, W., Schwarz, J., Jones, C., Liller, W., \& Fabian, A. C. 1979, ApJ, 234, L27

Georgakakis, A., Georgantopoulos, I., Stewart, G. C., Shanks, T., \& Boyle, B. J. 2003, MNRAS, 344, 161 
Georgakakis, A. E., Georgantopoulos, I., Basilakos, S., Plionis, M., \& Kolokotronis, V. 2004, MNRAS, 354, 123

Georgakakis, A. E., Chavushyan, V., Plionis, M., et al. 2006, MNRAS, 367, 1017

Georgakakis, A., Rowan-Robinson, M., Babbedge, T. S. R., \& Georgantopoulos, I. 2007, MNRAS, 377, 203

Georgantopoulos, I., Georgakakis, A., \& Koulouridis, E. 2005, MNRAS, 360, 782

Gilfanov, M. 2004, MNRAS, 349, 146

Gilfanov, M., Grimm, H.-J., \& Sunayev, R., 2004, MNRAS, 351, 1365

Griffiths, R. E., \& Padovani, P. 1990, ApJ, 360, 483

Grimm, H.-J., Gilfanov, M., \& Sunyaev, R. 2003, MNRAS, 339, 793

Helou, G., Soifer, B. T., \& Rowan-Robinson, M. 1985, ApJ, 298, L7

Ho, L. C., Filippenko, A. V., \& Sargent, W. L. W. 1997, ApJ, 112, 315

Hornschemeier, A. E., Heckman, T. E., Ptak, A. F., Tremonti, C. A., \& Colbert,

E. J. M. 2005, AJ, 129, 86

Hornschemeier, A. E., Bauer, F. E., Alexander, D. M., et al., 2003, ApJ, 126, 575

Irwin, M., Maddox, S., \& McMahon, R. G. 1994, Spectrum, 2, 14

Isobe, T., Feigelson, E. D., Akritas, M. G., \& Babu, G. J. 1990, ApJ, 364, 104

Kennicutt, R. C., \& Kent, S. M. 1983, AJ, 88, 1094

Kennicutt, R. C. Jr., Tamblyn, P., Congdon, C. E., et al. 1994, ApJ, 435, 22

Kewley, L. J., Geller, M. J., Jansen, R. A., \& Dopita, M. A. 2002, AJ, 124, 3135

Lasker, B. M., Doggett, J., McLean, B., et al. 1996, ASP Conf. Ser., 101, 88

Lehmann, I., Hasinger, G., Schmidt, M., et al. 2001, A\&A, 371, 833

Londish, D., Croom, S. M., Boyle, B. J., et al. 2002, MNRAS, 334, 941

Monet, D. G., Levine, S. E., Canzian, B., et al. 2003, ApJ, 125, 984

Moshir, M., Kopan, G., Cornow, T., et al. 1990, IRAS Faint Source Catalog (ver. 2.0; Greenbelt; NASA/GSFC)

Norman, C., Ptak, A., Hornschemeier, A., et al. 2004, ApJ, 607, 721
Owen, R. A., \& Warwick, R. S. 2009, MNRAS, in press [arXiv: 0901.4263]

Persic, M., \& Rephaeli, Y. 2007, A\&A, 463, 481

Persic, M., Rephaeli, Y., Braito, V., et al. 2004, A\&A, 419, 849

Ptak, A., Mobasher, B., Hornschemeier, A., Bauer, F., \& Norman, C. 2007, ApJ, 667,826

Ranalli, P., Comastri, A., \& Setti, G. 2003, A\&A, 399, 39

Read, A. M., \& Ponman, T. J. 2001, MNRAS, 328, 127

Read, A. M., Ponman, T. J., \& Strickland, D. K. 1997, MNRAS, 286, 626

Shapley, A., Fabbiano, G., \& Eskridge, P. B. 2001, ApJS, 137, 139

Skrutskie, M. F., Cutri, R. M., Stiening, R., et al. 2006, AJ, 131, 1163

Rosa-Gonzàlez, D., Terlevich, E., \& Terlevich, R. 2002, MNRAS, 332, 283

Savage, B. D., \& Mathis, J. S. 1979, ARA\&A, 17, 73

Sbarufatti, B., Treves, A., Falomo, R., et al. 2006, AJ, 132, 1

Stocke, J. T., Morris, S. L., Gioia, I. M., et al. 1991, ApJS, 76, 813

Tajer, M., Trinchieri, G., Wolter, A., et al. 2005, A\&A, 435, 799

Trinchieri, G., \& Fabbiano, G. 1985, ApJ, 296, 447

Trinchieri, G., Fabbiano, G., \& Bandiera, B. 1989, ApJ, 342, 759

Troitsky, S. 2008, MNRAS, 388, L79

Tyler, K., Quillen, A. C., LaPage, A., \& Rieke, G. H. 2004, ApJ, 610, 213

Tzanavaris, P., Georgantopoulos, I., \& Georgakakis, A. 2006, A\&A, 454, , 447

Tzanavaris, P., \& Georgantopoulos, I. 2008, A\&A, 480, 663

Veilleux, S., \& Osterbrock, D. E. 1987, ApJS, 63, 295

Vogler, A., Pietsch, W., \& Bertoldi, F. 1997, A\&A, 318, 768

Warwick, R. S., Jenkins, L. P., \& Read, A. M., 2007, MNRAS, 376, 1611

Watson, M. G., Pye, J. P., Denby, M., et al. 2003, AN, 324, 89

Zezas, A., 2000, Ph.D. Thesis, University of Leicester 
E. Rovilos et al.: Normal galaxies in the XMM-Newton fields, Online Material $p 1$

Table 2. X-ray, optical and near-infrared properties of the initial candidates.

\begin{tabular}{|c|c|c|c|c|c|c|c|c|c|c|}
\hline ID & XMM name & $z$ & 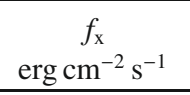 & $\begin{array}{l}\log L_{\mathrm{x}} \\
\mathrm{erg} \mathrm{s}^{-1} \\
\end{array}$ & $\begin{array}{c}R \\
\text { mag }\end{array}$ & $\log \frac{f_{\mathrm{x}}}{f_{\mathrm{o}}}$ & $\begin{array}{c}L_{\mathrm{H} \alpha} \\
\operatorname{erg~s}^{-1} \\
\end{array}$ & $\begin{array}{c}K \\
\text { mag }\end{array}$ & $\begin{array}{c}L_{\mathrm{K}} \\
\operatorname{erg~s}^{-1} \\
\end{array}$ & Type \\
\hline 1703 & J005818.3-355548 & $0.0489 \pm 0.0008$ & $6.421 \times 10^{-15}$ & 40.53 & 15.64 & -2.436 & 40.82 & 14.585 & 42.84 & emission \\
\hline 1774 & J005922.8-360933 & $0.1271 \pm 0.0007$ & $7.858 \times 10^{-15}$ & 41.51 & 16.27 & -2.099 & - & 13.982 & 43.95 & absorption \\
\hline 1778 & J005929.7-361113 & $0.0115 \pm 0.0003$ & $2.571 \times 10^{-14}$ & 39.87 & 12.38 & -3.140 & 41.50 & 14.042 & 41.81 & emission \\
\hline 3654 & J022416.7-050323 & - & $7.173 \times 10^{-15}$ & - & 15.38 & -2.492 & - & - & - & featureless \\
\hline 3716 & J022456.2-050801 & $0.0837 \pm 0.0008$ & $2.769 \times 10^{-14}$ & 41.67 & 12.57 & -3.032 & - & 13.839 & 43.63 & absorption \\
\hline 3773 & J022536.4-050012 & $0.0530 \pm 0.0002$ & $1.940 \times 10^{-14}$ & 41.11 & 12.40 & -3.252 & 41.76 & 13.298 & 43.45 & emission \\
\hline 3776 & J022537.8-050223 & - & $1.489 \times 10^{-14}$ & - & 13.94 & -2.751 & - & - & - & featureless \\
\hline 3777 & J022538.2-050806 & $0.0607 \pm 0.0003$ & $3.862 \times 10^{-15}$ & 40.53 & 15.67 & -2.645 & 41.17 & 15.670 & 42.61 & emission \\
\hline 3778 & J022538.3-050423 & 0.0000 & $1.157 \times 10^{-14}$ & STAR & 15.06 & -2.413 & - & - & - & STAR \\
\hline 4009 & J023613.5-523036 & $0.1117 \pm 0.0005$ & $8.520 \times 10^{-15}$ & 41.42 & 14.47 & -2.784 & 41.99 & 14.869 & 43.47 & emission \\
\hline 4298 & J030927.5-765223 & 0.0000 & $1.597 \times 10^{-14}$ & STAR & 14.99 & -2.301 & - & - & - & STAR \\
\hline 4379 & J031256.5-765039 & 0.0000 & $1.104 \times 10^{-14}$ & STAR & 13.12 & -3.209 & - & - & - & STAR \\
\hline 4481 & J031723.1-442056 & 0.0000 & $2.835 \times 10^{-14}$ & STAR & 14.82 & -2.119 & - & - & - & STAR \\
\hline 4736 & J031829.8-441140 & $0.0732 \pm 0.0005$ & $5.158 \times 10^{-14}$ & 41.82 & 12.19 & -2.914 & 42.25 & 14.366 & 43.30 & emission \\
\hline 4765 & J031845.0-441042 & $0.0735 \pm 0.0006$ & $8.163 \times 10^{-15}$ & 41.02 & 12.95 & -3.408 & 42.20 & 14.664 & 43.18 & emission \\
\hline 4927 & J033831.4-351421 & $0.139 \pm 0.003$ & $9.464 \times 10^{-14}$ & 42.66 & 13.31 & -2.202 & 40.85 & - & - & composite \\
\hline 5570 & J043306.5-610760 & $0.0589 \pm 0.0007$ & $1.304 \times 10^{-14}$ & 41.02 & 13.80 & -2.865 & - & 14.254 & 43.15 & absorption \\
\hline 5663 & J043333.5-612427 & $0.0604 \pm 0.0012$ & $1.382 \times 10^{-14}$ & 41.04 & 14.58 & -2.527 & - & 14.195 & 43.17 & absorption \\
\hline 7355 & J055940.7-503218 & $0.197 \pm 0.020$ & $8.546 \times 10^{-15}$ & 42.02 & 15.35 & -2.430 & - & 14.702 & 44.10 & absorption \\
\hline 7400 & J060014.9-502230 & - & $7.370 \times 10^{-15}$ & - & 15.56 & -2.411 & - & - & - & featureless \\
\hline 9062 & $\mathrm{~J} 124238.5-111919$ & - & $1.940 \times 10^{-14}$ & - & 13.45 & -2.832 & - & - & - & not observed \\
\hline 9064 & J124239.1-112822 & - & $8.434 \times 10^{-15}$ & - & 13.59 & -3.140 & - & - & - & not observed \\
\hline 9337 & J125204.5-292029 & - & $3.552 \times 10^{-14}$ & - & 13.81 & -2.426 & - & - & - & not observed \\
\hline 9480 & J125718.4-171441 & - & $1.182 \times 10^{-14}$ & - & 12.21 & -3.543 & - & - & - & not observed \\
\hline 9539 & J125819.1-171837 & - & $1.512 \times 10^{-14}$ & - & 12.12 & -3.472 & - & - & - & not observed \\
\hline 9730 & J133032.4-014735 & - & $5.251 \times 10^{-15}$ & - & 14.05 & -3.162 & - & - & - & not observed \\
\hline 9966 & $\mathrm{~J} 133527.7-342630$ & - & $1.014 \times 10^{-14}$ & - & 15.15 & -2.434 & - & - & - & not observed \\
\hline 12281 & J201329.7-414737 & $0.1293 \pm 0.0006$ & $1.078 \times 10^{-14}$ & 41.67 & 14.69 & -2.591 & 43.49 & 14.047 & 43.93 & emission \\
\hline 12308 & $\mathrm{~J} 201345.0-563713$ & $0.0541 \pm 0.0005$ & $1.114 \times 10^{-14}$ & 40.89 & 13.49 & -3.057 & - & 12.947 & 43.61 & absorption \\
\hline 13137 & $\mathrm{~J} 213758.7-143611$ & $0.0524 \pm 0.0002$ & $4.446 \times 10^{-15}$ & 40.46 & 14.08 & -3.222 & 41.84 & 13.829 & 43.22 & emission \\
\hline 14252 & $\mathrm{~J} 221543.0-173958$ & - & $2.929 \times 10^{-15}$ & - & 17.44 & -2.057 & - & - & - & not observed \\
\hline 14402 & $\mathrm{~J} 221726.0-082531$ & $0.0845 \pm 0.0002$ & $2.733 \times 10^{-14}$ & 41.67 & 13.97 & -2.477 & - & 13.759 & 43.67 & absorption \\
\hline 14541 & $\mathrm{~J} 222110.0-244749$ & - & $8.297 \times 10^{-15}$ & - & 16.06 & -2.157 & - & - & - & featureless \\
\hline 14608 & J222804.4-051751 & - & $5.319 \times 10^{-15}$ & - & 15.03 & -2.762 & - & - & - & featureless \\
\hline 14640 & $\mathrm{~J} 222818.3-050745$ & - & $7.741 \times 10^{-15}$ & - & 15.44 & -2.435 & - & - & - & not observed \\
\hline 14683 & J222834.0-052818 & - & $9.300 \times 10^{-15}$ & - & 13.88 & -2.980 & - & - & - & featureless \\
\hline 15022 & $\mathrm{~J} 225149.3-175225$ & $0.0776 \pm 0.0005$ & $1.711 \times 10^{-14}$ & 41.39 & 14.12 & -2.621 & 42.59 & 13.744 & 43.59 & emission \\
\hline 15393 & $\mathrm{~J} 231421.6-424559$ & $0.0672 \pm 0.0006$ & $2.039 \times 10^{-14}$ & 41.35 & 12.47 & -3.203 & - & 13.391 & 43.63 & absorption \\
\hline 15584 & $\mathrm{~J} 231851.8-423114$ & $0.11432 \pm 0.00015$ & $1.460 \times 10^{-14}$ & 41.68 & 13.74 & -2.840 & - & 13.985 & 43.85 & absorption \\
\hline 15631 & $\mathrm{~J} 232454.9-120459$ & $0.0809 \pm 0.0005$ & $1.164 \times 10^{-14}$ & 41.27 & 15.81 & -2.110 & 40.30 & - & - & composite \\
\hline 15867 & $\mathrm{~J} 235340.6-102420$ & $0.0742 \pm 0.0011$ & $1.972 \times 10^{-14}$ & 41.42 & 12.15 & -3.347 & - & 13.985 & 43.47 & absorption \\
\hline 15892 & $\mathrm{~J} 235405.7-101829$ & $0.0739 \pm 0.0006$ & $7.687 \times 10^{-15}$ & 40.99 & 13.38 & -3.262 & - & 13.780 & 43.53 & absorption \\
\hline 15926 & J235418.1-102013 & $0.0804 \pm 0.0003$ & $1.349 \times 10^{-14}$ & 41.32 & 14.48 & -2.580 & 43.38 & 14.040 & 43.51 & emission \\
\hline 15997 & $\mathrm{~J} 235629.1-343743$ & $0.0523 \pm 0.0009$ & $1.437 \times 10^{-14}$ & 40.96 & 12.12 & -3.494 & - & 13.136 & 43.49 & absorption \\
\hline
\end{tabular}


E. Rovilos et al.: Normal galaxies in the XMM-Newton fields, Online Material $p 2$
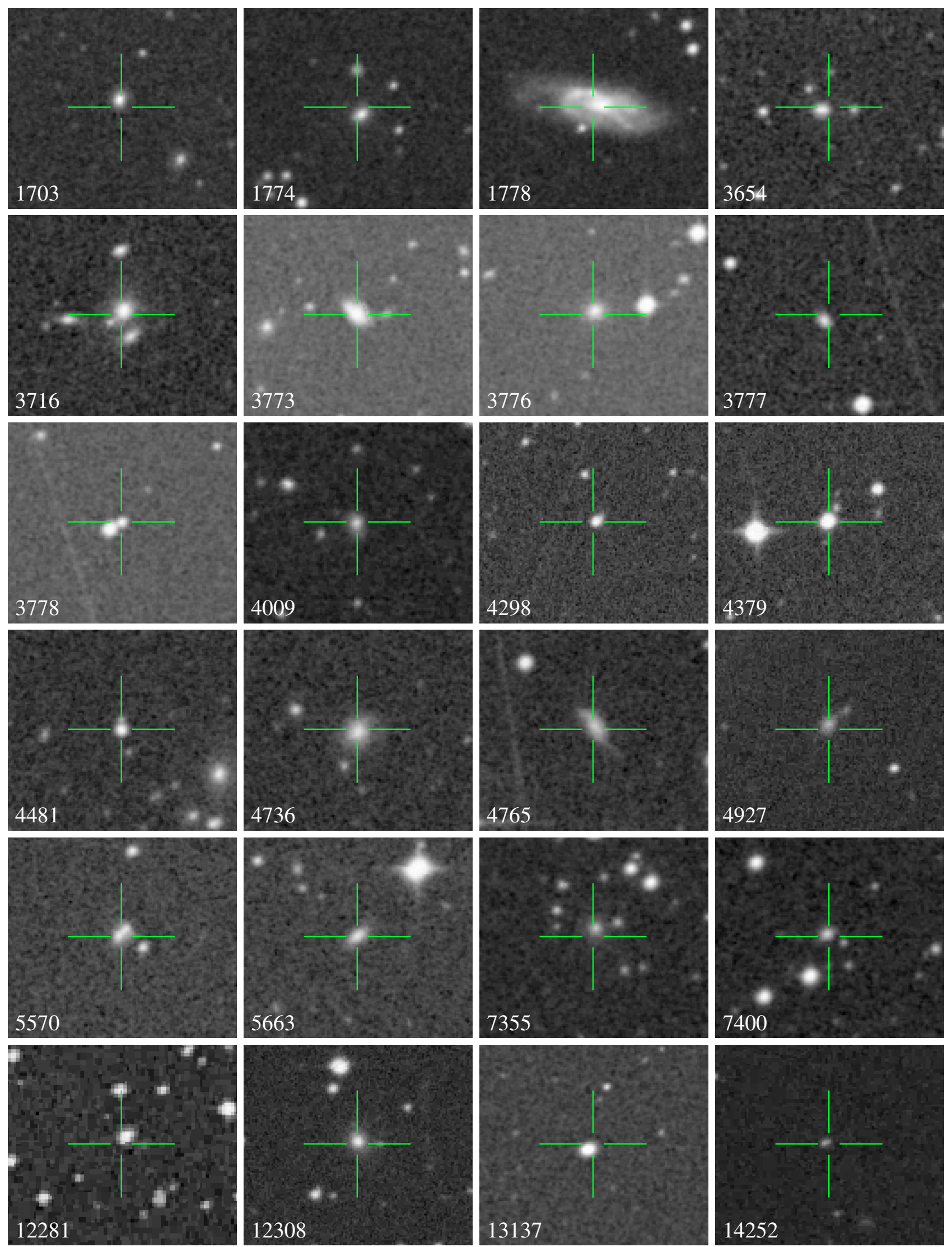

Fig. 1. Optical $(R)$ images of the normal galaxy candidates, centred on the X-ray position. The inner radii of the crosses are 6 arcsec and the outer 30 arcsec. 
E. Rovilos et al.: Normal galaxies in the XMM-Newton fields, Online Material $p 3$
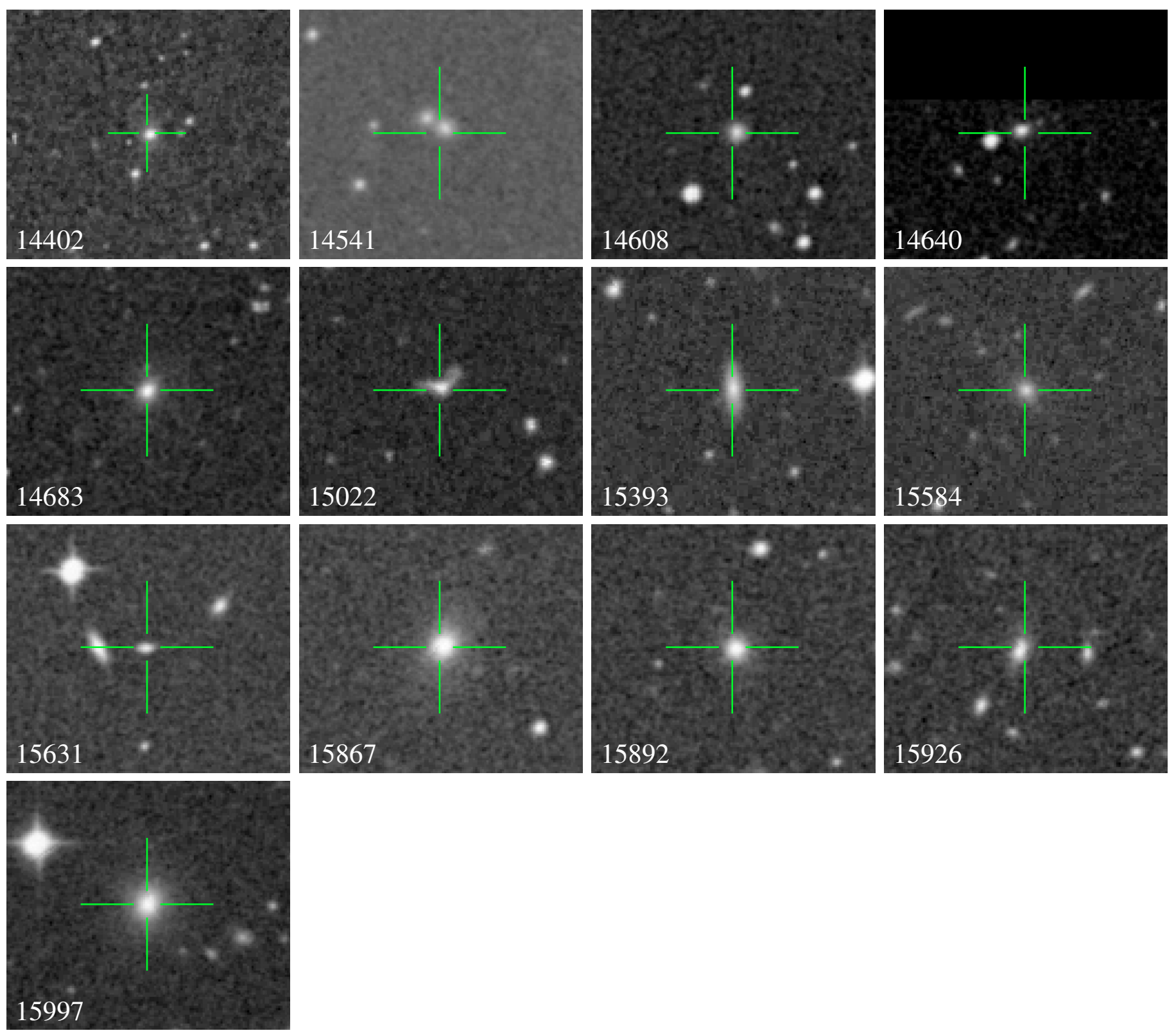

Fig. 1. continued. 
E. Rovilos et al.: Normal galaxies in the XMM-Newton fields, Online Material $p 4$
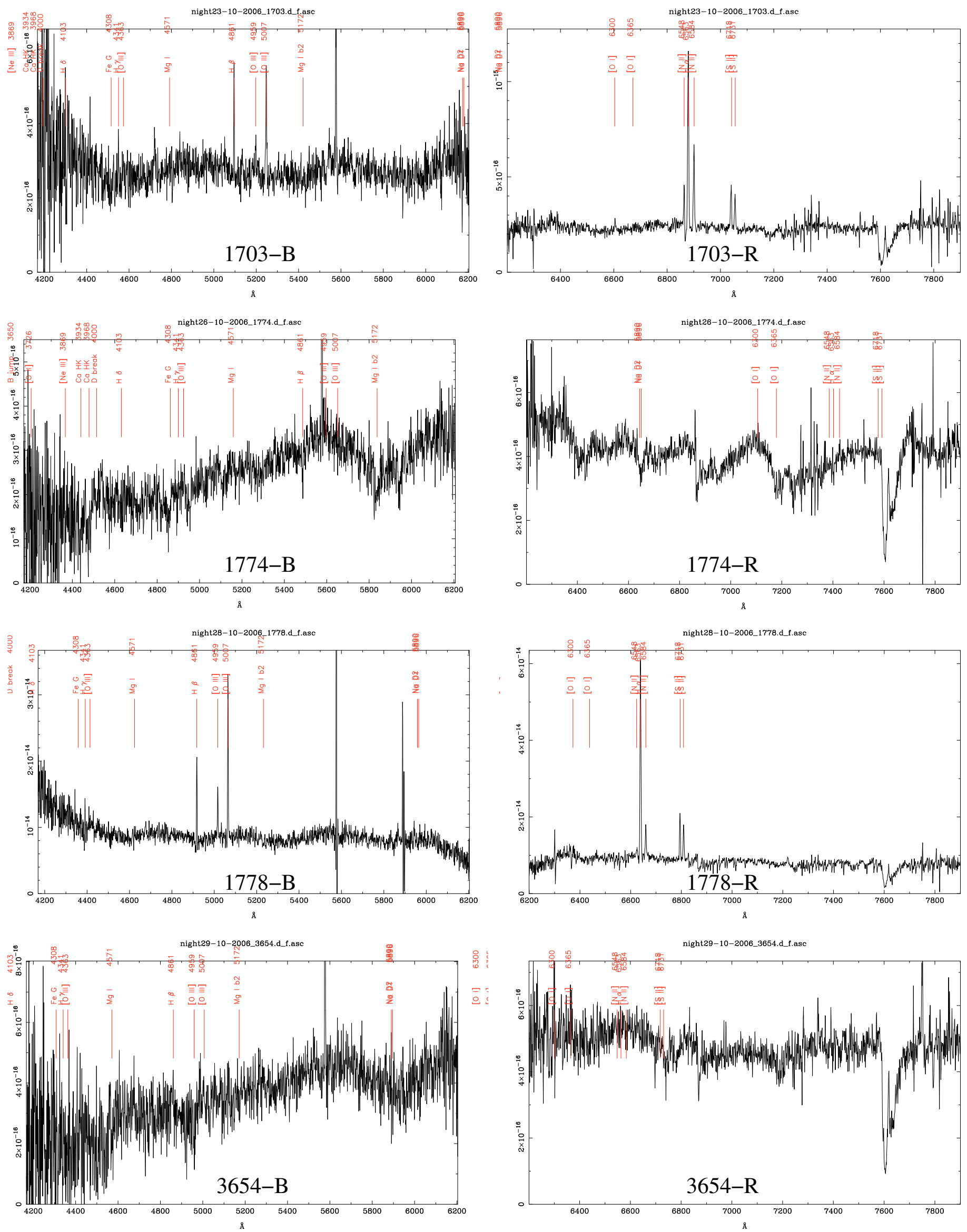

Fig. 3. Optical spectra of the normal galaxy candidates. 
E. Rovilos et al.: Normal galaxies in the XMM-Newton fields, Online Material p 5
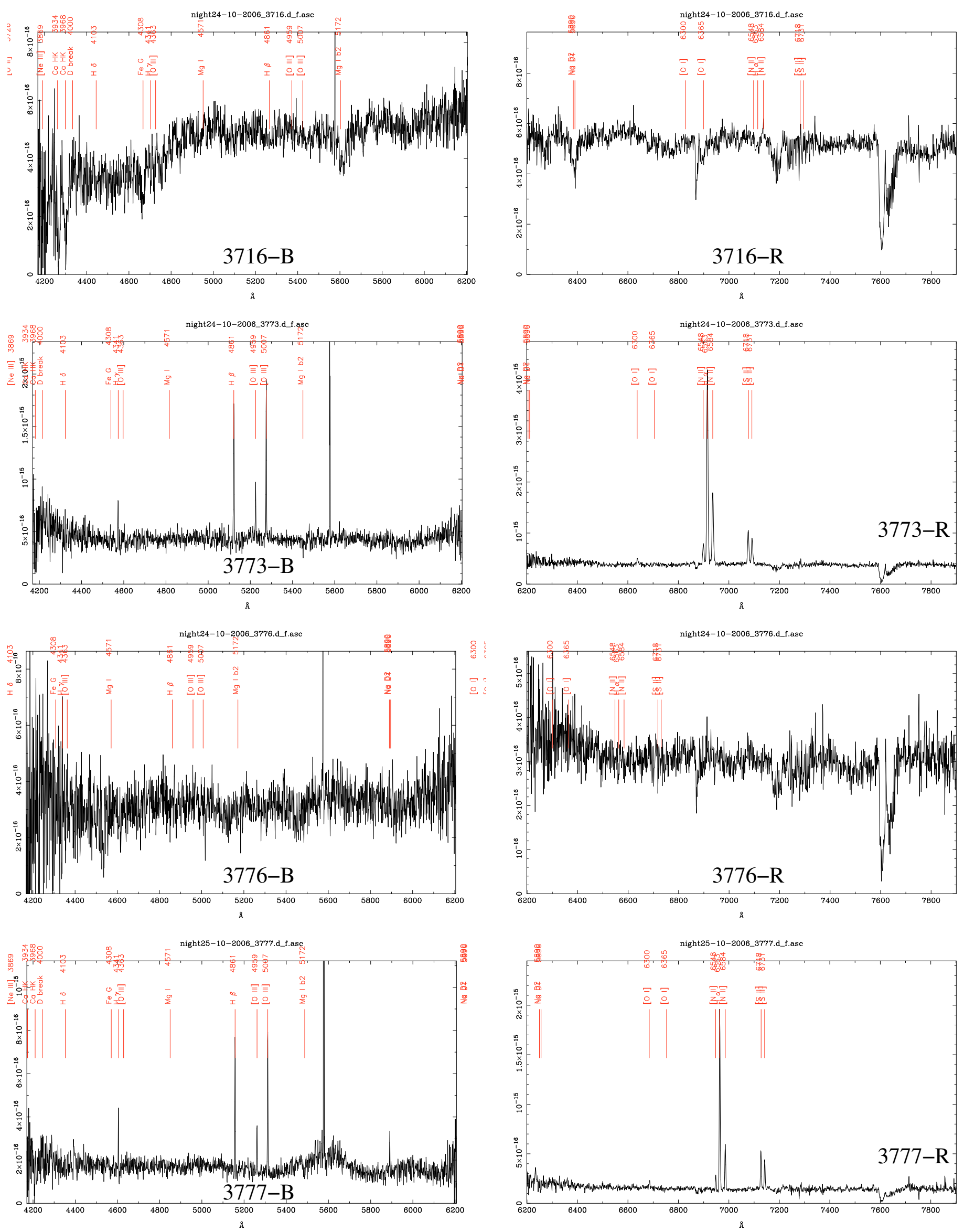

Fig. 3. continued. 
E. Rovilos et al.: Normal galaxies in the XMM-Newton fields, Online Material $p 6$
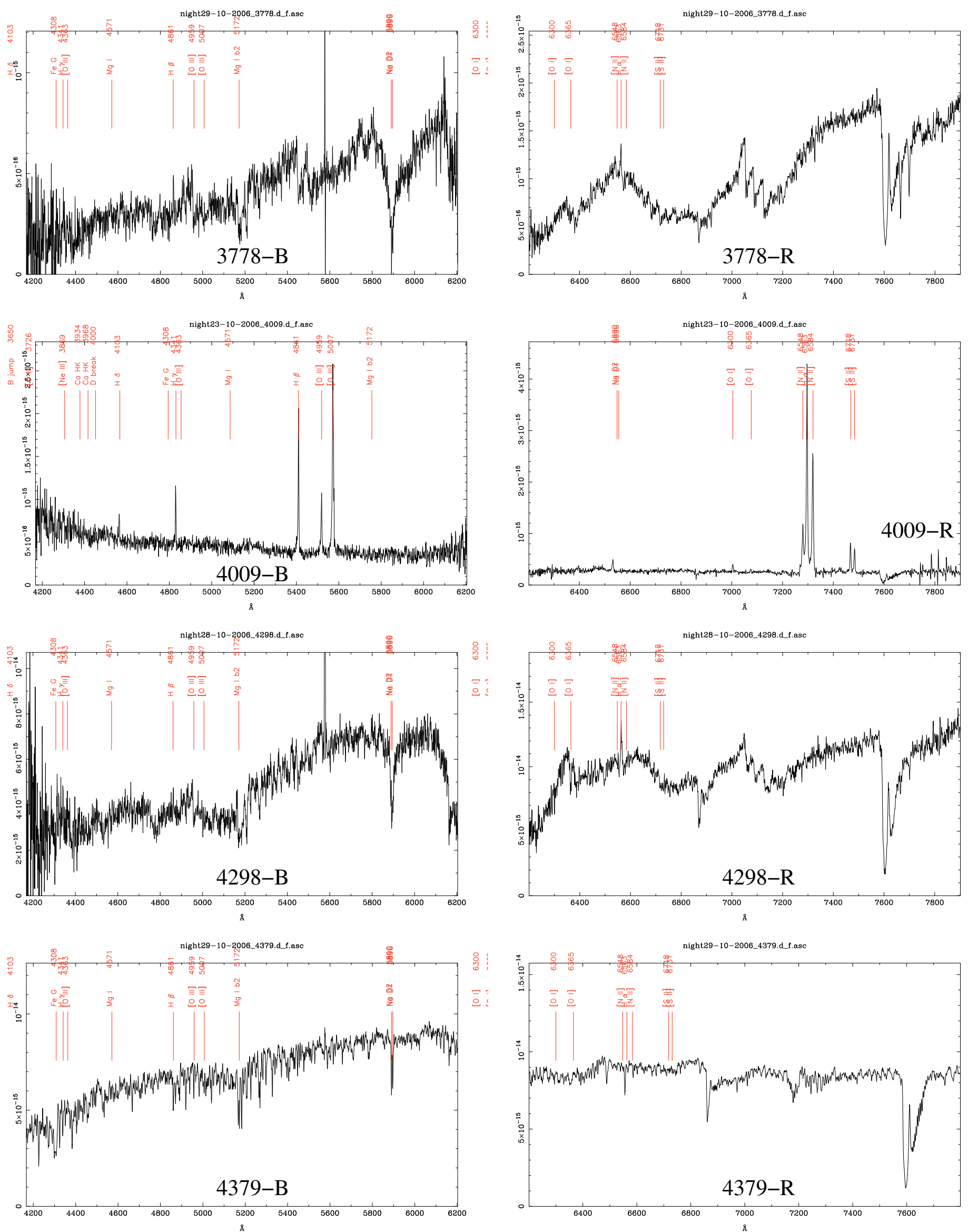

Fig. 3. continued. 
E. Rovilos et al.: Normal galaxies in the XMM-Newton fields, Online Material $p 7$
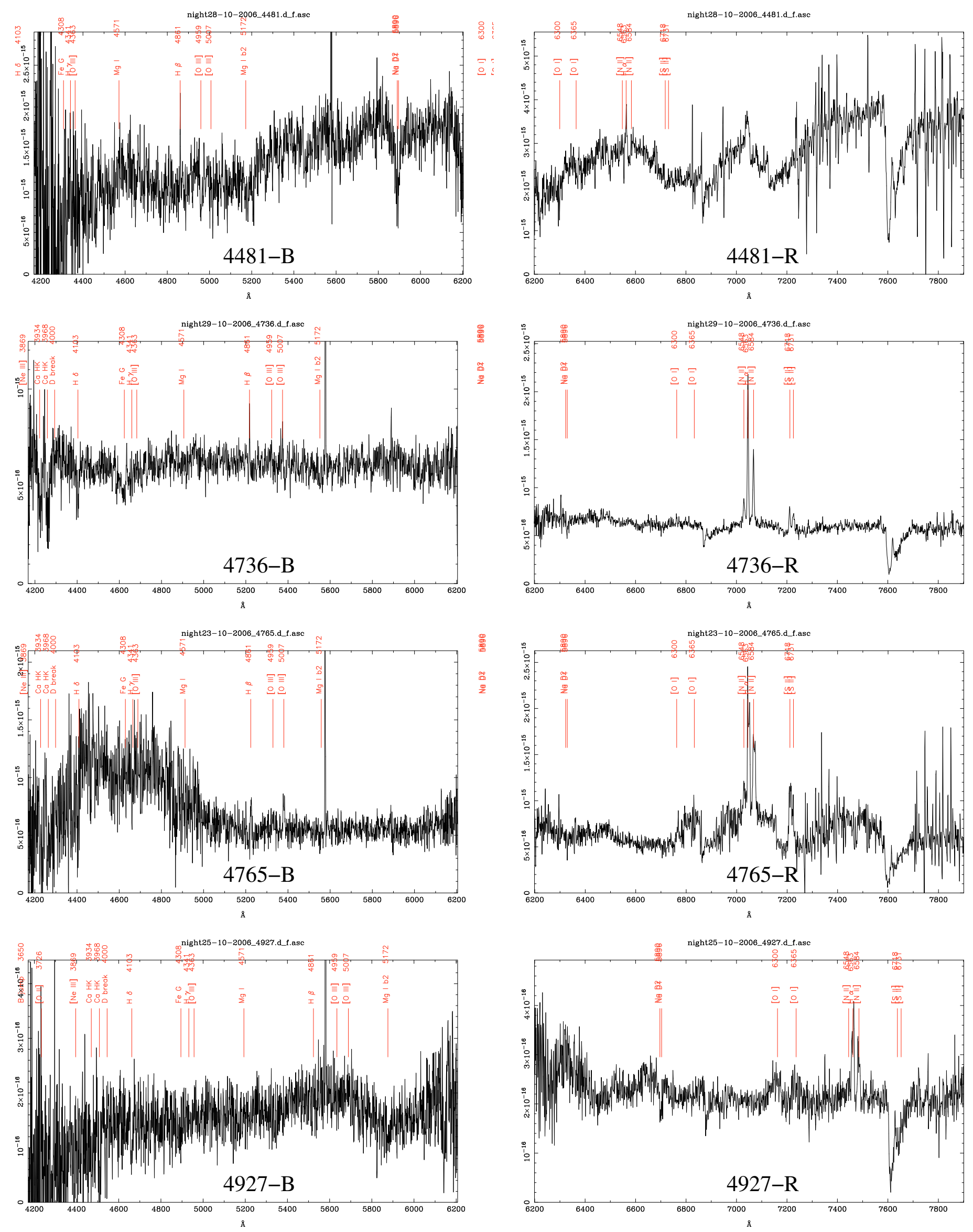

Fig. 3. continued. 
E. Rovilos et al.: Normal galaxies in the XMM-Newton fields, Online Material $p 8$
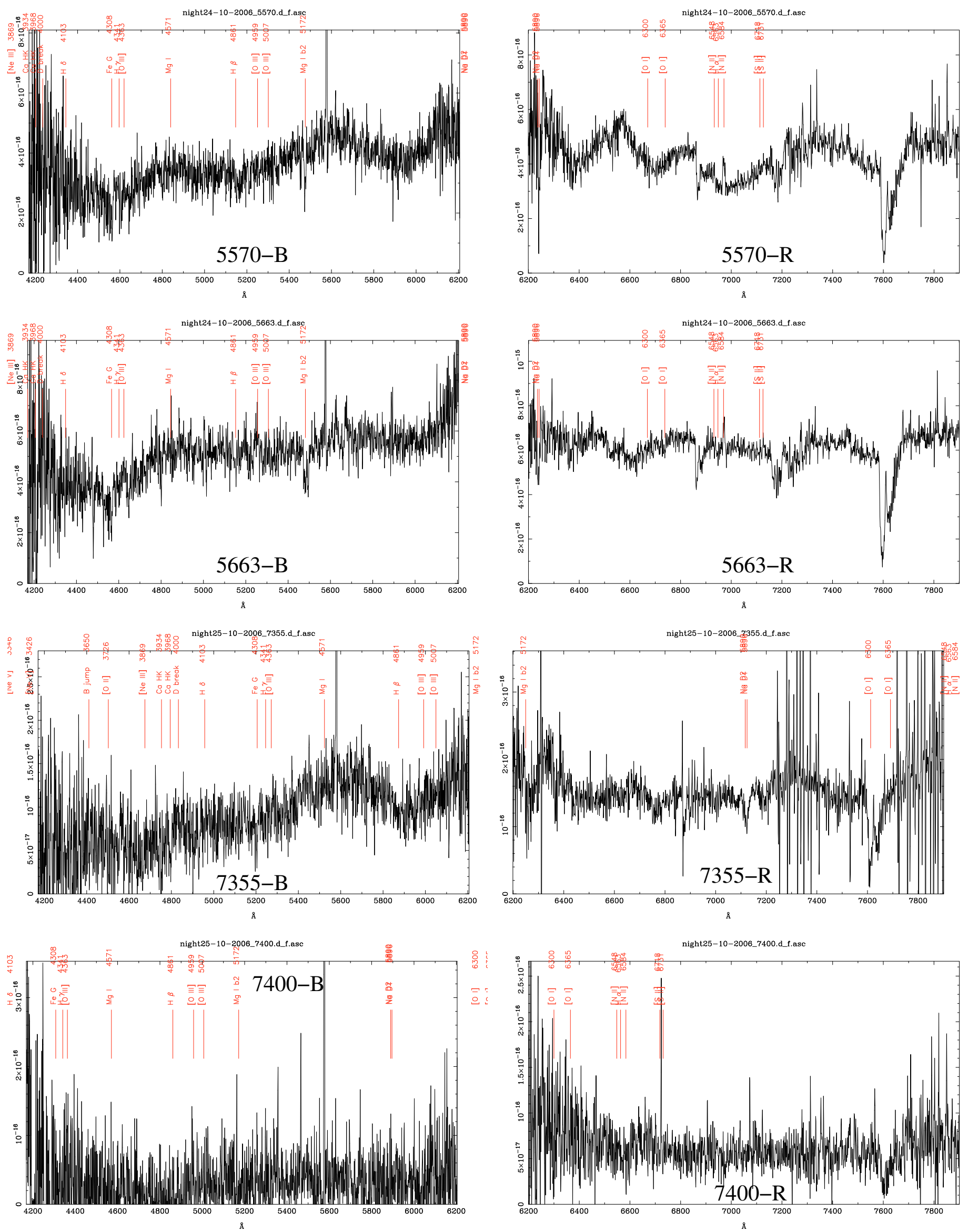

Fig. 3. continued. 
E. Rovilos et al.: Normal galaxies in the XMM-Newton fields, Online Material $p 9$
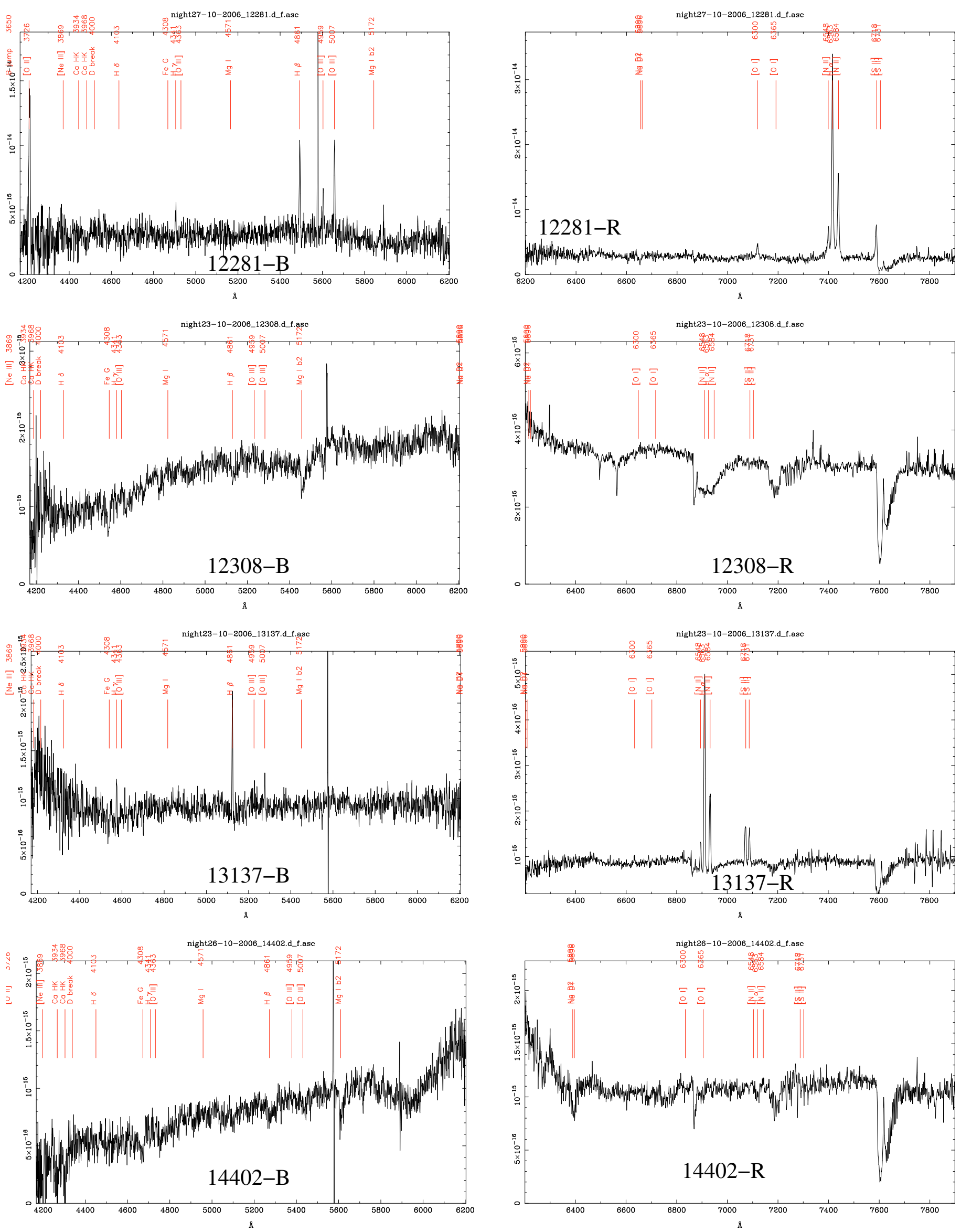

Fig. 3. continued. 
E. Rovilos et al.: Normal galaxies in the XMM-Newton fields, Online Material p 10
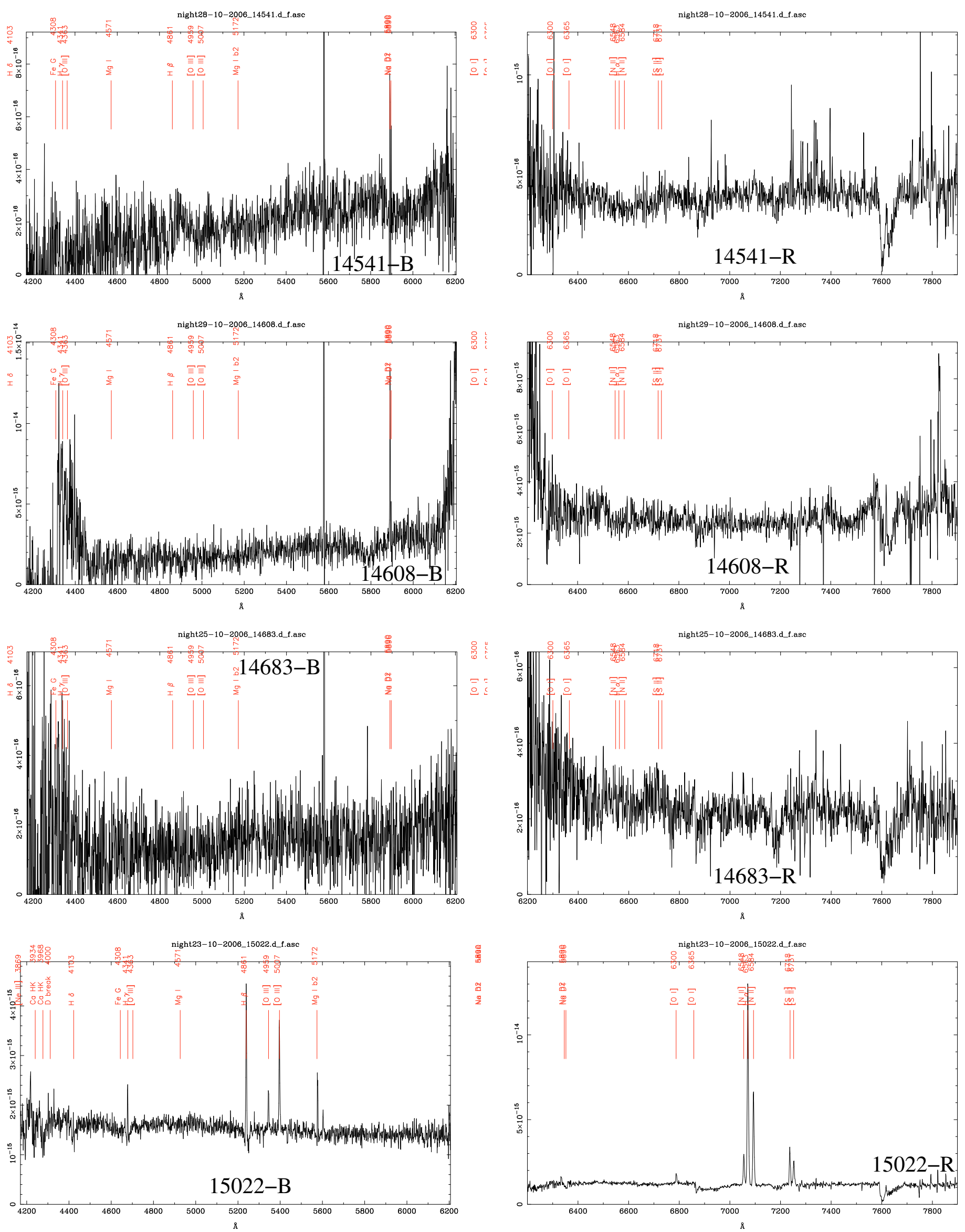

Fig. 3. continued. 
E. Rovilos et al.: Normal galaxies in the XMM-Newton fields, Online Material $p 11$
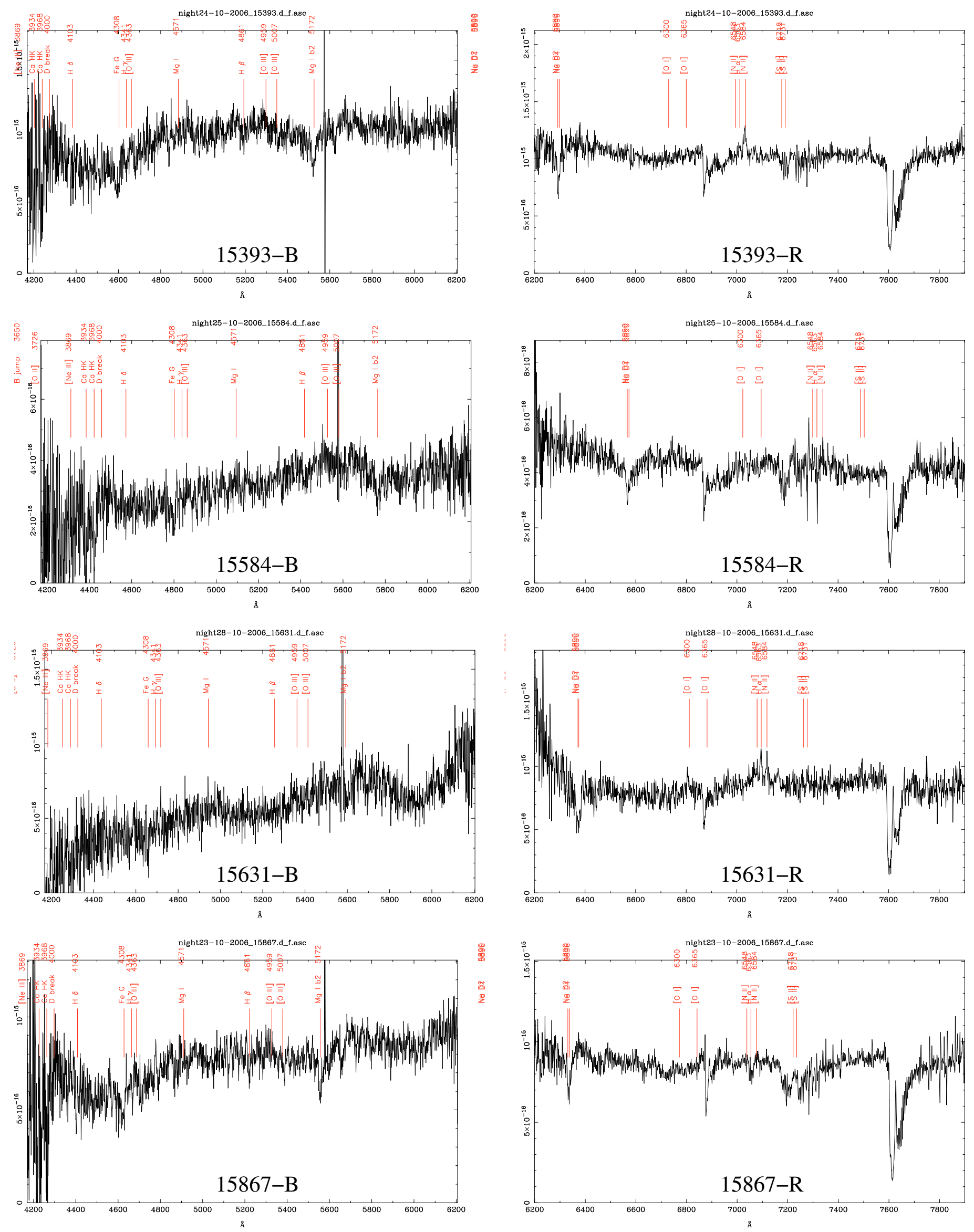

Fig. 3. continued. 
E. Rovilos et al.: Normal galaxies in the XMM-Newton fields, Online Material p 12
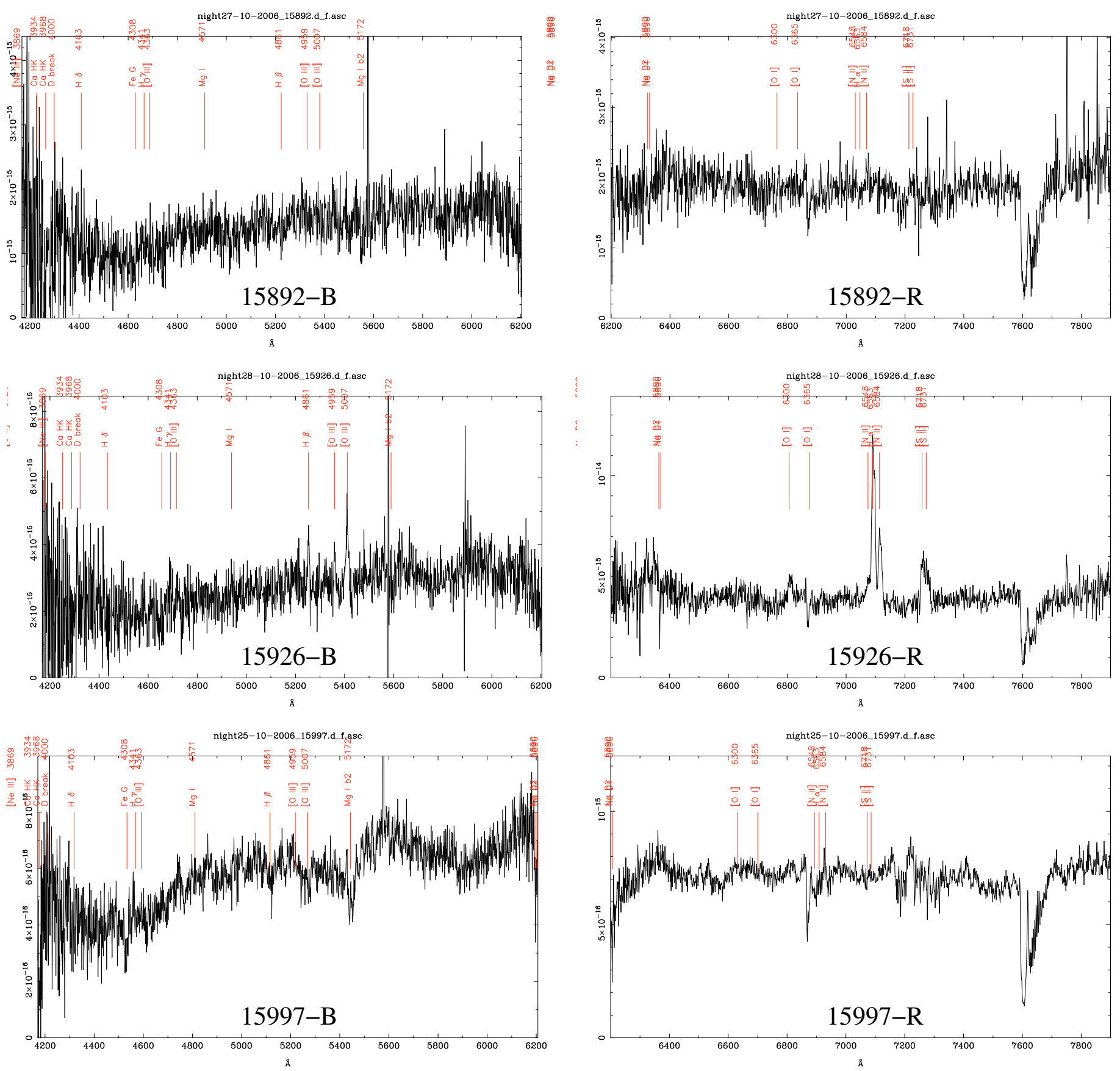

Fig. 3. continued. 